\title{
Erratum to: Revisiting the global electroweak fit of the Standard Model and beyond with Gfitter
}

\author{
H. Flächer ${ }^{1}$, M. Goebel ${ }^{2,3}$, J. Haller ${ }^{3}$, A. Hoecker ${ }^{1, a}$, K. Mönig ${ }^{2}$, J. Stelzer ${ }^{2}$ \\ ${ }^{1}$ CERN, Geneva, Switzerland \\ ${ }^{2}$ DESY, Hamburg and Zeuthen, Germany \\ ${ }^{3}$ Institut für Experimentalphysik, Universität Hamburg, Hamburg, Germany
}

Received: 5 July 2011 / Published online: 12 August 2011

(C) Springer-Verlag / Società Italiana di Fisica 2011

\section{Erratum to: Eur. Phys. J. C (2009) 60:543-583 DOI 10.1140/epjc/s10052-009-0966-6}

Our above-mentioned publication requires changes of citations in the description of the global electroweak fit of the Standard Model and corrections of mistakes in the results of the fit of the two Higgs doublet model (2HDM). We emphasise in particular the use of ZFITTER implementations [1, 2] for the calculation of the partial and total widths of the $Z$ and of the total width of the $W$ boson. The following changes should be made.

Replacement of the sixth sentence in the fourth paragraph in Sect. 1 "Introduction" The sentence should be replaced by "The calculations of the partial and total widths of the $Z$ and of the total width of the $W$ boson have been integrated from the ZFITTER package [1, 2] into the Gfitter subpackage GSM and are co-authored by both groups [3]. ${ }^{1} \mathrm{It}$ includes up to two-loop electroweak corrections [1, 2, 4-13] and all known QCD corrections [1, 2, 14]."

Correction of $R_{b}^{0}$ constraints For the theoretical prediction of the electroweak precision observable $R_{b}^{0}$ in the 2HDM we used Eqs. (6.3) and (6.4) from Ref. [15]. These equations contained a misprint and the common factors $e /\left(s_{W} c_{W}\right)$ should be removed. We are grateful to Pietro Slavich and Giuseppe Degrassi for drawing our attention to this. After correction we obtain slightly tighter exclusion regions for

${ }^{1}$ Usage and citation of the Gfitter subpackage GSM should include a citation of the ZFITTER package [1,2].

The online version of the original article can be found under doi:10.1140/epjc/s10052-009-0966-6.

a e-mail: andreas.hoecker@cern.ch
$R_{b}^{0}$ as shown in Fig. 1. This figure replaces Fig. 5.1 (top, left) of our publication. The correction will correspondingly affect the combined exclusion regions drawn in Figs. 5.2 of the publication, which is, however, hardly visible due to the large range in $\tan \beta$ shown in these plots. Since our paper QCD corrections to $R_{b}^{0}$ have been published [16] that will be included in a future update of our analysis.

Typo correction of (5.3) The correct formula should read

$r_{H}=\left(1-m_{B}^{2} \frac{\tan ^{2} \beta}{M_{H^{ \pm}}^{2}}\right)^{2}$.

Replacement of the first three sentences in Sect. A.3 "Electroweak form factors" The sentences should be replaced by "The electroweak form factors for lepton or quark flavours $f, \rho_{Z}^{f}$ and $\kappa_{Z}^{f}$, absorbing the radiative corrections, are used in the Gfitter software for the calculation of the partial and total widths of the $Z$ boson and of the total width of the $W$ boson. The relevant implementations have been integrated from the ZFITTER package [1, 2] (cf. Footnote 1) and are co-authored by both groups [3]. It includes up to two-loop electroweak corrections [1, 2, 4-13] and all known QCD corrections [1, 2, 14]. In these calculations the intermediate on-shell mass scheme [2] is used, which lies between OMS-I and OMS-II."

Replacement of the third sentence in Sect. A.4 "Radiator functions" The sentence should be replaced by "The following formulae as implemented in the Gfitter subpackage GSM are taken from [13] and the ZFITTER package $[1,2]$ (cf. Footnote 1). The relevant implementations are coauthored by both groups [3]." 
Fig. 1 Updated two-sided 68\%, 95\% and $99 \%$ CL $2 \mathrm{HDM}$ exclusion regions obtained from $R_{b}^{0}$.

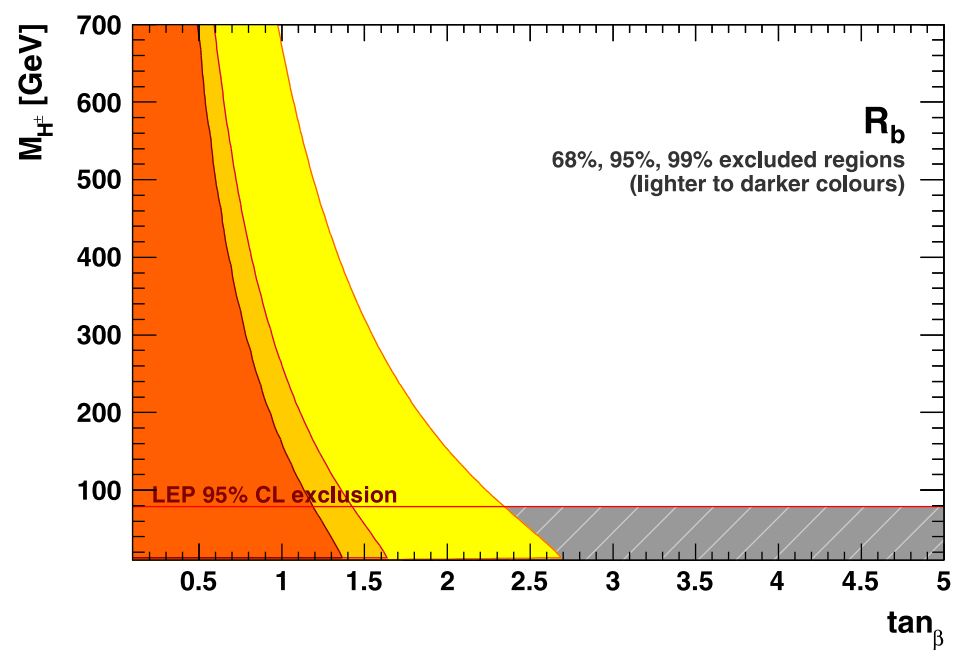

\section{References}

1. A.B. Arbuzov et al., Comput. Phys. Commun. 174, 728 (2006). hep-ph/0507146, http://zfitter.desy.de

2. D.Y. Bardin et al., Comput. Phys. Commun. 133, 229 (2001). hep-ph/9908433, http://zfitter.desy.de

3. A. Akhundov, A. Arbuzov, M. Awramik, D. Bardin, M. Bilenky, P. Christova, M. Czakon, O. Fedorenko, A. Freitas, M. Goebel, M. Gruenewald, J. Haller, A. Hoecker, L. Kalinovskaya, K. Moenig, A. Olchevsky, S. Riemann, T. Riemann, Gfitter/GSM sub-package, public release in preparation

4. A.A. Akhundov, D.Y. Bardin, T. Riemann, Nucl. Phys. B 276, 1 (1986)

5. D.Y. Bardin, S. Riemann, T. Riemann, Z. Phys. C 32, 121 (1986)

6. R. Barbieri, M. Beccaria, P. Ciafaloni, G. Curci, A. Vicere, Nucl. Phys. B 409, 105 (1993)

7. J. Fleischer, O.V. Tarasov, F. Jegerlehner, Phys. Lett. B 319, 249 (1993)

8. G. Degrassi, S. Fanchiotti, F. Feruglio, B.P. Gambino, A. Vicini, Phys. Lett. B 350, 75 (1995). hep-ph/9412380
9. G. Degrassi, F. Feruglio, A. Vicini, S. Fanchiotti, P. Gambino, hep-ph/9507286

10. G. Degrassi, P. Gambino, A. Vicini, Phys. Lett. B 383, 219 (1996). hep-ph/9603374

11. G. Degrassi, P. Gambino, Nucl. Phys. B 567, 3 (2000). hep-ph/9905472

12. Electroweak working group, D.Y. Bardin et al., hep-ph/9709229. Prepared for Workshop Group on Precision Calculations for the $\mathrm{Z}$ Resonance (2nd meeting held Mar 31, 3rd meeting held Jun 13), Geneva, Switzerland, 14 Jan 1994, CERN-YELLOW-95-03A

13. D.Y. Bardin, G. Passarino, The Standard Model in the Making: Precision Study of the Electroweak Interactions (Clarendon, Oxford, 1999) $685 \mathrm{pp}$.

14. B.A. Kniehl, Nucl. Phys. B 347, 86 (1990)

15. H.E. Haber, H.E. Logan, Phys. Rev. D 62, 015011 (2000). hep-ph/9909335

16. G. Degrassi, P. Slavich, Phys. Rev. D 81, 075001 (2010). 1002.1071 